\title{
Preparation of Metal-Free Nitrogen-Doped Carbon Material and Its Catalytic Performance
}

\author{
Xuan Wang, Lei Yang, Ke Ying Cai*, Ying Mei Zhou, Peng Wang, Ming Song \\ School of Chemistry and Chemical Engineering, Xuzhou University of Technology, Xuzhou, Jiangsu \\ 221018, China
}

Received: 1st May 2018; Revised: 30th September 2018; Accepted: $2^{\text {nd }}$ October 2018; Available online: $25^{\text {th }}$ January 2019; Published regularly: April 2019

\begin{abstract}
Nitrogen-doped carbon materials (NCMs) were prepared via hydrothermal treatment together with pyrolysis under nitrogen atmosphere by using melamine as nitrogen source and sucrose as carbon source. The NCMs were characterized by X-ray diffraction (XRD), laser Raman spectroscopy and X-ray photoelectron spectroscopy (XPS). The results showed that nitrogen species were successfully doped into NCMs in the form of pyridinic N, pyrrolic N, graphitic N, and oxidized N. With the temperature of pyrolysis increasing, the total amount of nitrogen species decreased, while the proportion of graphitic $\mathrm{N}$ increased. The catalytic performance was investigated by the reduction of $p$-nitrophenol with excessive $\mathrm{KBH}_{4}$ at $30{ }^{\circ} \mathrm{C}$. The reaction rate constant can reach $1.06 \mathrm{~min}^{-1}$ for NCM-800. The NCM-800 has good stability, which can be used for 8 cycles without obvious deactivation. Copyright (C) 2018 BCREC Group. All rights reserved
\end{abstract}

Keywords: $p$-Nitrophenol; Nitrogen-Doped Carbon Materials; Catalytic Reduction

How to Cite: Wang, X., Yang, L., Cai, K.Y., Zhou, Y.M., Wang, P., Song, M. (2019). Preparation of Metal-Free Nitrogen-Doped Carbon Material and Its Catalytic Performance. Bulletin of Chemical Reaction Engineering \& Catalysis, 14 (1): 105-111 (doi:10.9767/bcrec.14.1.2593.105-111)

Permalink/DOI: https://doi.org/10.9767/bcrec.14.1.2593.105-111

\section{Introduction}

Carbon material has a large specific surface area and rich pore structure, so it is commonly used as an adsorbent [1,2]. The hydrophilicity of carbon material is not very well, which limits its application. The doping of non-metallic elements, such as $\mathrm{O}, \mathrm{N}$, and $\mathrm{B}$, attracts increasing attention as an interesting and effective method for modifying and creating chemical functions. The way of doping heteroatom can change the charge distribution on the surface of carbon material and enhance its ability to adsorb ions or

* Corresponding Author.

E-mail: caikeying@163.com (K.Y. Cai)

Telp: +86-13852081673, Fax: +86-516-85608300 polar molecules, thus expanding the scope of its application [3,4].

Nitrogen atoms can easily replace carbon atoms due to their similar radii. Since the charge distribution in nitrogen-doped carbon materials $(\mathrm{NCMs})$ is uneven, its ability of adsorbing metal particles is enhanced, and it can be served as a good catalyst carrier for various metals. There are many ways to prepare $\mathrm{NCMs}$, for instance, CVD method, solvothermal method, epitaxial growth method, arc discharge method, flame method, hydrothermal method, and so on [5]. Usually, NCMs can be used as supercapacitor electrode materials, catalysts for oxygen reduction reaction (ORR), and catalyst supports. As catalyst supports, NCMs can improve the sur- 
face charge density of metal particles, such as: Co [6] and $\mathrm{Ru}$ [7], which facilitates the catalytic activity. Recent studies indicate that NCMs can be directly used as catalysts for some heterogeneous catalytic reactions, such as: dehydrogenation of ethylbenzene [8], the catalytic reductions of nitrobenzene [9] or phenylacetylene [10], catalytic oxidation of ethylbenzene to acetophenone [11], and catalytic hydrogen chlorination of acetylene [12]. Compared with metal-loaded NCMs, metal-free NCMs would not suffer from the aggregation and loss of metal particles, which are certain disadvantages for metallic catalysts [13]. Therefore, as metal-free catalysts, NCMs have some advantages, for instance, low cost and good stability.

$p$-Nitrophenol is an intermediate raw material that is commonly used in chemical synthesis, but it is high toxic and not easy to degrade in the environment [14]. On the contrary, $p$-aminophenol, the reduction substance of $p$-nitrophenol, is less toxic and easy to degrade. Hence, the catalytic reduction of $p$-nitrophenol to $p$-aminophenol has been extensively studied. Some of catalysts for the reduction of $p$-nitrophenol are loaded with precious metals as active components, such as: gold [15] and silver [16]. Non-precious metals can also be loaded in the material as active components, such as: cobalt/hydrotalcite [17] and copper/perlite [18].

NCMs can be directly used for catalytic reduction of $p$-nitrophenol with $\mathrm{NaBH}_{4}$ [9], but most of the preparations are of too much trouble. In this work, a facile method was used to prepare NCMs with melamine and sucrose as raw materials via hydrothermal method and pyrolysis. The NCMs served as metal-free catalysts showed excellent catalytic performance in the reduction of $p$-nitrophenol.

\section{Materials and Methods}

\subsection{Reagents and Instruments}

Melamine and $\mathrm{KBH}_{4}$ (95 $w t \%$ ) purchased from Sinopharm Chemical Reagent Co. Ltd. (China) were of analytical pure grade. Sucrose (95 $w t \%)$ was purchased from supermarket. $p$ Nitrophenol purchased from Shanghai Qiangshun Chemical Reagent Co. Ltd. (China) was of analytical pure grade.

\subsection{Preparation and Characterization of Cata- lysts}

NCMs were fabricated by hydrothermal reaction and pyrolysis of melamine and sucrose. In a typical procedure, melamine $(8 \mathrm{~g})$, sucrose
$(2 \mathrm{~g})$ and $30 \mathrm{~mL}$ of deionized water were added into a beaker and stirred for $30 \mathrm{~min}$ at room temperature. The obtained mixture was directly transferred into a $50 \mathrm{~mL}$ of Teflon-lined autoclave, and heated to $200{ }^{\circ} \mathrm{C}$ for $8 \mathrm{~h}$. After washed with alcohol and deionized water, the resulting solid was further heated at a rate of $10{ }^{\circ} \mathrm{C} / \mathrm{min}$ to $800{ }^{\circ} \mathrm{C}$ for $2 \mathrm{~h}$ in a tubular furnace under nitrogen atmosphere. The as-prepared sample was marked as NCM-800. The samples treated at $700{ }^{\circ} \mathrm{C}$ and $900{ }^{\circ} \mathrm{C}$ with the same process were marked NCM-700 and NCM-900, respectively.

The X-ray diffraction (XRD) patterns were recorded on a diffractometer (Rigaku Ultima IV) equipped with a $\mathrm{Cu}-K_{\mathrm{a}}$ radiation source. It was operated under the following conditions: tube voltage $40 \mathrm{kV}$, current $40 \mathrm{~mA}, \lambda=0.15406$ $\mathrm{nm}$, and scanning range $2 \theta=10-80^{\circ}$. The chemical compositions of the as-prepared samples were obtained with an X-ray Photoelectron Spectrometer (XPS, Thermo ESCALAB 250XI) using Al- $K_{a} \mathrm{X}$-ray as the excitation source (225 W). Raman spectra were recorded by a Raman spectrometer (Thermo Fisher Scientific DXR2) using $532 \mathrm{~nm}$ incident. The morphology and microstructure of the as-prepared samples were investigated with Transmission Electron Microscope (TEM, JEOL JEM-2100).

\subsection{Catalytic Reduction of $p$-nitrophenol}

The catalytic performance of the asprepared NCMs was investigated by using the reduction of $p$-nitrophenol with $\mathrm{KBH}_{4}$ as a model reaction. Briefly, $95 \mathrm{~mL}$ of deionized water and $5 \mathrm{~mL}$ of $p$-nitrophenol $(40 \mathrm{mmol} / \mathrm{L})$ aqueous solution was added into a $250 \mathrm{~mL}$ beaker. The temperature of the solution was kept at $30{ }^{\circ} \mathrm{C}$ and stirred. Then $0.31 \mathrm{~g}$ of $\mathrm{KBH}_{4}$ $(8 \mathrm{mmol})$ and $0.03 \mathrm{~g}$ of NCM catalyst were added into the solution. Samples were directly withdrawn from the reaction mixture at certain time intervals.

In alkaline conditions, the characteristic absorption wavelength of $p$-nitrophenol and $p$ aminophenol are about $400 \mathrm{~nm}$ and $300 \mathrm{~nm}$, respectively. With the consumption of $p$ nitrophenol, the absorbance at $400 \mathrm{~nm}$ decreases gradually, and the continuous production of $p$-aminophenol leads to the gradual increase of the absorbance at $300 \mathrm{~nm}[9,19] \mathrm{si}$ multaneously. Therefore, the reaction progress can be directly monitored by a UV-vis spectrometer (Shanghai Metash UV-5500PC). After the reaction completed, the catalyst was recovered by centrifugalization, washed, and reused repeatedly. 


\section{Results and Discussion}

\subsection{Characterization of the Catalysts}

The phase of the as-prepared samples was examined by XRD. For all the three samples, the peaks at about $25^{\circ}$ and $44^{\circ}$ of the patterns shown in Figure 1 were attributed to the (002) and (100) diffraction planes of graphitic carbon (JCPDS No. 41-1487) [20], respectively. The diffraction peaks in the three patterns are relatively wide, which indicate the low degree of graphitization of the materials.

Raman spectroscopy is a good technique to detect the structure and electronic properties of NCMs. To further analyze these samples, the three Raman spectra of the NCM samples are illustrated in Figure 2. As it can be seen from Figure 2, two peaks at 1350 and $1580 \mathrm{~cm}^{-1}$ are observed from the NCM samples. D band at $1350 \mathrm{~cm}^{-1}$ is mainly ascribed to the defects and disorders in the crystal lattice. $\mathrm{G}$ band at 1580 $\mathrm{cm}^{-1}$ is ascribed to the in-plane vibrational mode of the $\mathrm{sp}^{2}$ hybridized carbon atoms. Both $\mathrm{D}$ band and $\mathrm{G}$ band indicate the presence of crystalline graphite carbon [4,21]. All the three spectra show broad D bands, which indicate that all the three samples have a large degree of disorder [7]. Furthermore, the intensity ratio of $\mathrm{D}$ band $\left(I_{D}\right)$ and $\mathrm{G}$ band $\left(I_{G}\right)$ is usually used to evaluate the structural defects of carbon materials brought by nitrogen-doping. The larger the $I_{G} / I_{D}$ ratio is, the higher the degree of graphitization of carbon material is [22,23]. The $I_{G} / I_{D}$ ratios of NCM-700, NCM-800, and NCM-900 are 0.92, 0.93, and 0.97, respectively, indicating that NCM-900 has higher degree of graphitization. The results of Raman spectra are consistent with the results of XRD characterization.

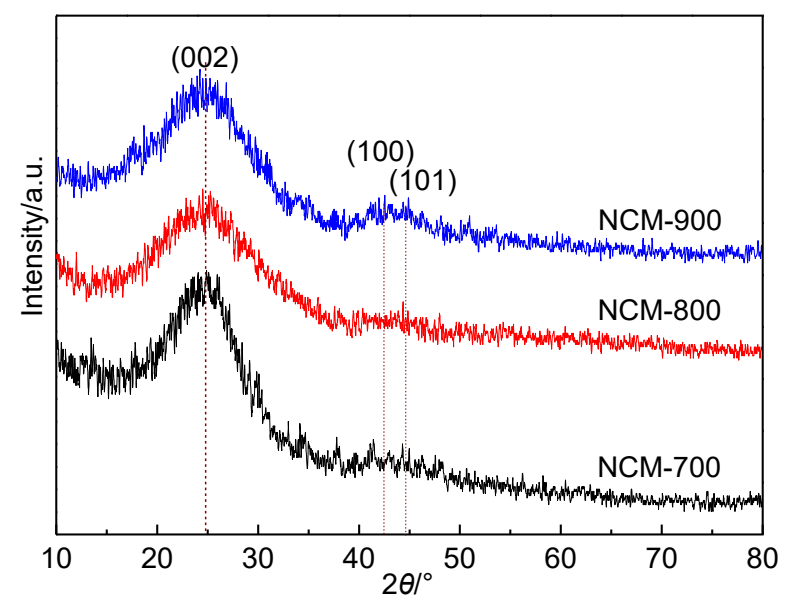

Figure 1. XRD patterns of the NCMs
XPS is a common powerful tool to detect different $\mathrm{N}$ species by different bind energies. The three XPS spectra (Figure 3a) indicate that the NCMs contained $\mathrm{C}, \mathrm{N}$, and $\mathrm{O}$ elements. That is, $\mathrm{N}$ was successfully doped in the materials. The N 1s high-resolution spectra of the three NCMs samples are deconvoluted into four single peaks (Figure 3b-d), corresponding to pyridinic $\mathrm{N}(398.04 \mathrm{eV})$, pyrrolic $\mathrm{N}(400.25 \mathrm{eV})$, graphitic N (401.09 eV) and oxidized N (403.2 $\mathrm{eV})[4,7,24]$. The contents of various forms of nitrogen species were calculated from different areas of the peaks (Table 1). As it can be seen from Table 1, with the increase of pyrolyzation temperature, the content of total nitrogen decreases, but the proportion of graphitic $\mathrm{N}$ increases, which is consistent with the results of related references [25,26]. It is supposed that graphitic $\mathrm{N}$ appears higher thermal stability.

The morphology was characterized by TEM. As shown in Figure 4, it is clear that there are wrinkled and transparent layers in the NCM800 sample. The results indicate that graphene-like material was prepared successfully.

\subsection{Catalytic Activity}

The as-prepared NCMs are used as catalysts for the reduction of $p$-nitrophenol with excessive $\mathrm{KBH}_{4}$. Figure 5a indicates the timedependent UV-Vis absorption spectra changed for the reduction process with NCM-800 as the catalyst. When the catalyst was introduced into the reaction solution, the absorption peak at $400 \mathrm{~nm}$ significantly decreases within $4 \mathrm{~min}$, meanwhile, a new peak at $300 \mathrm{~nm}$ appeared. This result indicates that $p$-nitrophenol was reduced to $p$-aminophenol [19]. Due to excessive $\mathrm{KBH}_{4}$ during the reaction, this reduction can be regarded as a first order reaction [18].



Figure 2. Raman spectra of the NCMs 
Hence, the kinetic equation for the reduction can be written as Equation (1).

$$
\ln \left(A / A_{0}\right)=\ln \left(c / c_{0}\right)=-k t
$$

According to the slope of the straight line, the reaction rate constant $k$ can be obtained from Figure 5b. As it can be seen from Figure 5b, the reaction rate constants of NCM-700, NCM-800 and NCM-900 are 0.13, 1.06, and $0.44 \mathrm{~min}^{-1}$, respectively. NCM-800 appears the highest catalytic activity, but NCM-700 appears almost no catalytic activity.

Although NCM-700 possesses the highest content of $\mathrm{N}$, its activity is the lowest among the three NCM samples, which is inconsistent with the normal conclusion of good effects re-

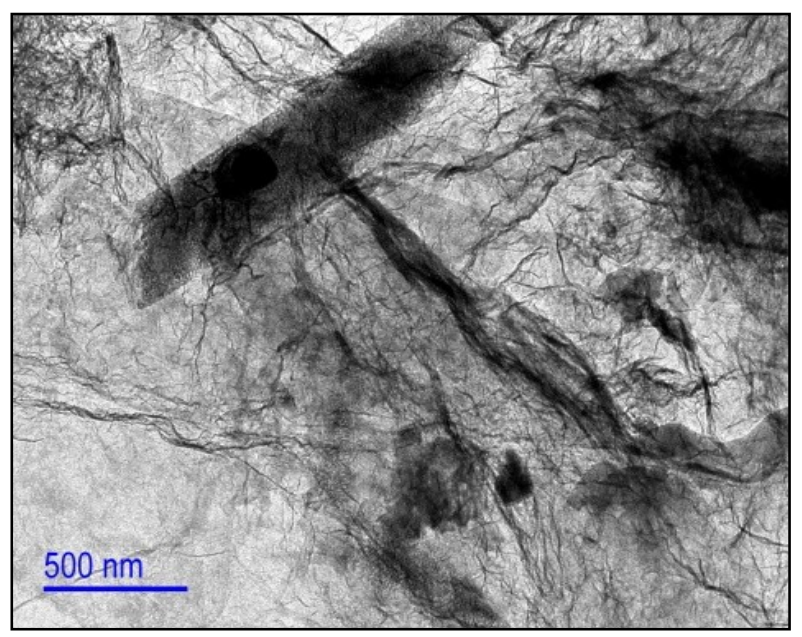

Figure 4. TEM image of NCM-800
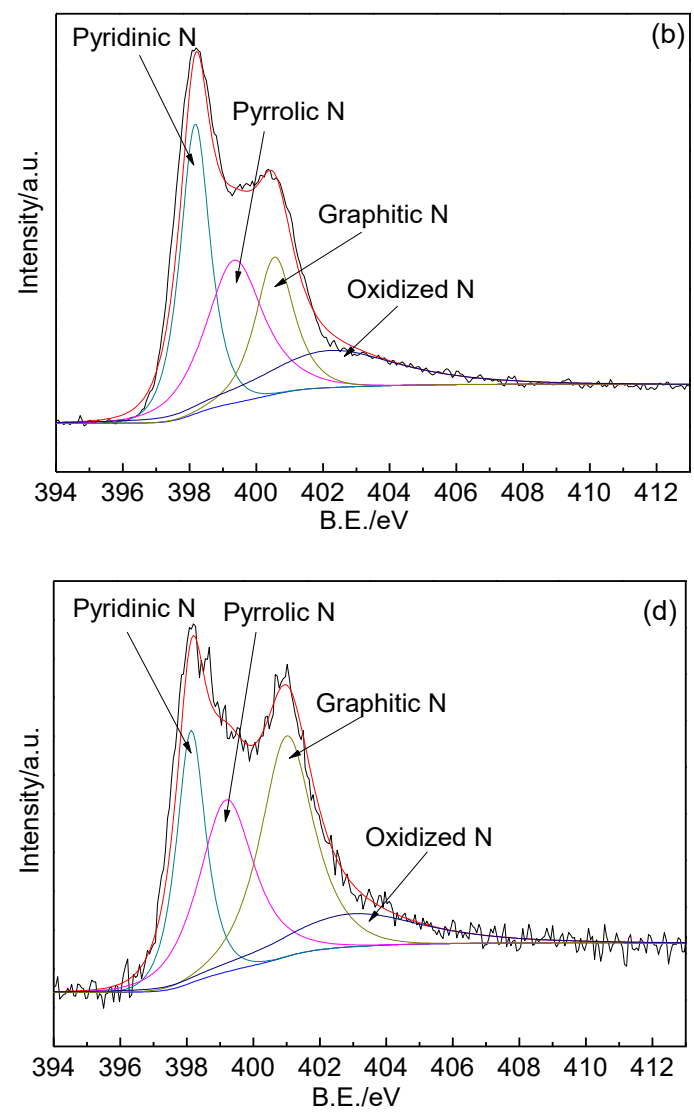

Figure 3. (a) XPS survey scan. High-resolution of N1s spectrum of (b) NCM-700, (c) NCM-800, and (d) NCM-900

Table 1. Deconvolution of N1s Spectra of NCMs

\begin{tabular}{cccccc}
\hline \multirow{2}{*}{ Samples } & \multirow{2}{*}{ N (\%) } & \multicolumn{4}{c}{ Nitrogen species (\%) } \\
\cline { 3 - 6 } & & Pyridinic & Pyrrolic & Graphitic & Oxidized \\
\hline NCM-700 & 19.54 & 31.88 & 30.36 & 19.42 & 18.34 \\
NCM-800 & 12.29 & 33.06 & 27.95 & 21.53 & 17.46 \\
NCM-900 & 9.56 & 22.26 & 28.72 & 35.14 & 13.88 \\
\hline
\end{tabular}


sulting from the large amount of $\mathrm{N}[11,27]$. The increased content of $\mathrm{N}$ atoms can cause topological defects leading to the decrease in the conductivity of the NCMs in some way, which affects the catalytic activity of NCM [22,28]. The surface chemistry and the surface structures including defects, graphitic edge and vacancies notably affect the properties of catalytically active sites. The appropriate amounts of surface defects, graphitic edges and vacancies are essential for the reaction [29]. NCM-900 has the highest degree of graphitization, but its total nitrogen content is the lowest of the three NCM samples, so its catalytic activity is not very high. Since both the graphitization degree and $\mathrm{N}$ atoms work for the catalytic performance, NCM-800 with appropriate content of N and graphitization degree exhibits highest catalytic activity.

\subsection{Reusability}

The reusability of NCM-800 is shown in Figure 6 . It can be reused for 8 cycles without obvious deactivation. This result indicates that the NCM-800 catalyst was not deactivated significantly during the reaction, washing, and separation processes. Compared with a metal-based catalyst, since no metal particle was loaded on the NCM, there is no aggregation or loss of metal particles during the reaction, which facilitates the stability of the NCM catalyst in the reaction. However, the activity of the catalyst decreased slightly after each use, which may be ascribed to the loss of NCM particles during their separation from the solution.

\section{Conclusions}

The NCMs were prepared by hydrothermal reaction and pyrolysis of melamine and sucrose. As a metal-free catalyst, the NCM, pyrolyzed at $800{ }^{\circ} \mathrm{C}$, has appropriate proportion of $\mathrm{N}$ and graphitization degree, which leads to higher catalytic activity and better stability for the reduction of $p$-nitrophenol with excess $\mathrm{KBH}_{4}$. The reaction rate constant with NCM800 as the catalyst can reach $1.06 \mathrm{~min}^{-1}$ at 30 ${ }^{\circ} \mathrm{C}$, and NCM-800 catalyst can be reused for 8 cycles without obvious deactivation.

\section{Acknowledgments}

The authors wish to acknowledge the financial supports from the Science and Technology Department of Jiangsu Province (BE2015041 and BK20171169), and Jiangnan University (3516010241140190/002).

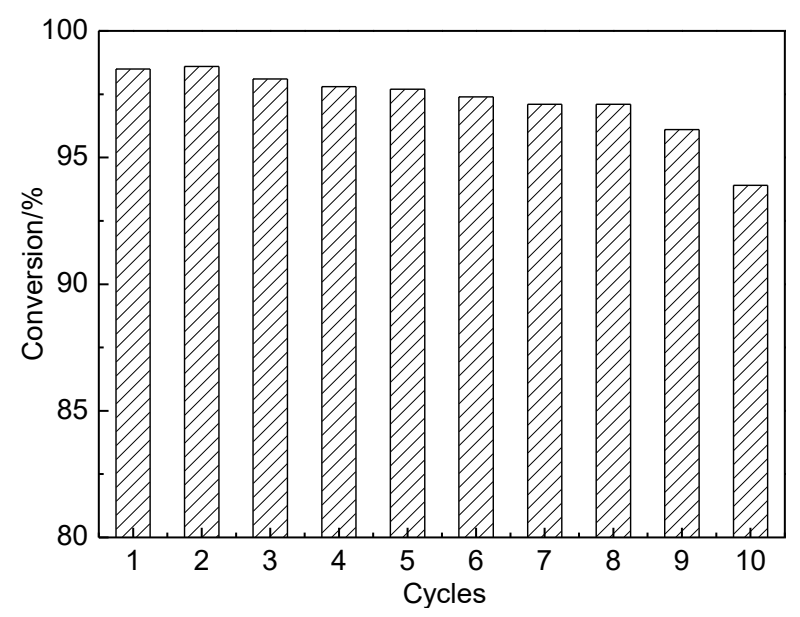

Figure 6. Reusability of NCM- 800

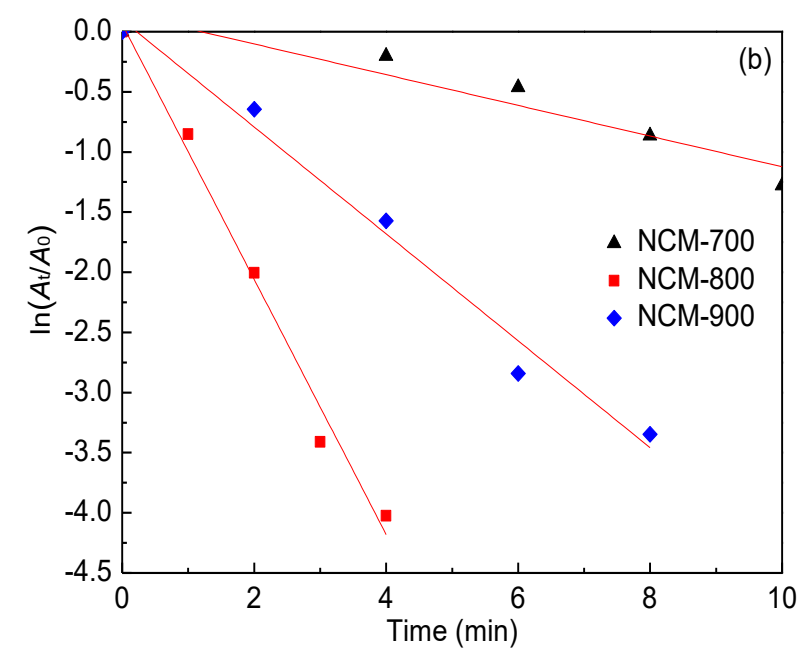

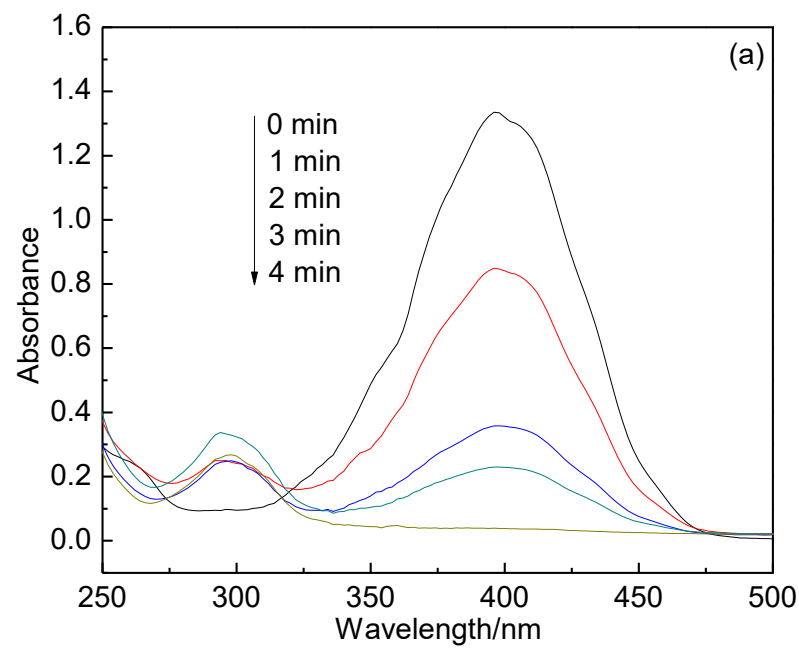

(a)

Figure 5. (a) Successive UV-vis absorption spectra of the reduction of 4-nitrophenol in the presence of NCM-800. (b) Plot of $\ln \left(A_{t} / A_{0}\right)$ against reaction time 


\section{References}

[1] Wang, J., Kaskel, S. (2012). KOH Activation of Carbon-Based Materials for Energy Storage. Journal of Materials Chemistry. 22(45): 23710-23725.

[2] Wu, F.C., Tseng, R.L., Hu, C.C., Wang, C.C. (2006). The Capacitive Characteristics of Activated Carbons-Comparisons of the Activation Methods on the Pore Structure and Effects of the Pore Structure and Electrolyte on the Capacitive Performance. Journal of Power Sources. 159(2): 1532-1542.

[3] Li, L.X., Zhang, Y.Q., Sun, P.S., An, B.G., Xing, T.Y., Song, R.F. (2016). Preparation of Pt-loaded Nitrogen-Doped Activated Aarbons and their Aatalytic Activities for the Oxygen Reduction Reaction. Carbon. 31(3): 287-292.

[4] Liu, L., Lu, J., Zhang, Y.X., Liu, M., Yu, Y.F., Chen, A.B. (2017). Synthesis of NitrogenDoped Graphitic Carbon Nanocapsules from a Poly (Ionic Liquid) for $\mathrm{CO}_{2}$ Capture. New Carbon Materials. 32(4): 380-384.

[5] Fan, M., Feng, Z.Q., Zhu, C., Chen, X., Chen, C., Yang, J., Sun, D. (2016). Recent Progress in 2D or 3D N-Doped Graphene Synthesis and the Characterizations, Properties, and Modulations of N Species. Journal of Materials Science. 51(23): 10323-10349.

[6] Xu, J., Gao, P., Zhao, T.S. (2012). NonPrecious $\mathrm{Co}_{3} \mathrm{O}_{4}$ Nano-Rod Electrocatalyst for Oxygen Reduction Reaction in AnionExchange Membrane Fuel Cells. Energy \& Environmental Science. 5(1): 5333-5339.

[7] Weijie, G., Shujing, G., Hongbo, Z., Xiulian, P., Xinhe, B. (2011). Enhanced Ammonia Synthesis Activity of Ru Supported on NitrogenDoped Carbon Nanotubes. Chinese Journal of Catalysis. 32(8): 1418-1423.

[8] Duong-Viet, C., Ba, H., Liu, Y., Truong-Phuoc, L., Nhut, J.M., Pham-Huu, C. (2014). Nitrogen-Doped Carbon Nanotubes on Silicon Carbide as a Metal-free Catalyst. Chinese Journal of Catalysis. 35(6): 906-913.

[9] Liu, J., Yan, X., Wang, L., Kong, L., Jian, P. (2017). Three-Dimensional Nitrogen-Doped Graphene Foam as Metal-Free Catalyst for the Hydrogenation Reduction of $p$ Nitrophenol. Journal of Colloid and Interface Science. 497: 102-107.

[10] Fujita, S.I., Asano, S., Arai, M. (2016). Nitrobenzene-Assisted Reduction of Phenylacetylene with Hydrazine over Nitrogen-Doped Metal-Free Activated Carbon Catalyst: Significance of Interactions Among Substrates and Catalyst. Journal of Molecular Catalysis A: Chemical. 423: 181-184.
[11] Tang, P., Gao, Y., Yang, J., Li, W., Zhao, H., Ma, D. (2014). Growth Mechanism of NDoped Graphene Materials and their Catalytic Behavior in the Selective Oxidation of Ethylbenzene. Chinese Journal of Catalysis. 35(6): 922-928.

[12] Zhou, K., Li, B., Zhang, Q., Huang, J.Q., Tian, G.L., Jia, J.C., Wei, F. (2014). The Catalytic Pathways of Hydrohalogenation Over Metal-Free Nitrogen-Doped Carbon Nanotubes. Chemsuschem. 7(3): 723-728.

[13] Ombaka, L.M., Ndungu, P., Nyamori, V.O. (2013). Usage of Carbon Nanotubes as Platinum and Nickel Catalyst Support in Dehydrogenation Reactions. Catalysis Today. 217(5): 65-75.

[14] Dong, Z., Le, X., Li, X., Zhang, W., Dong, C., Ma, J. (2014). Silver Nanoparticles Immobilized on Fibrous Nano-Silica as Highly Efficient and Recyclable Heterogeneous Catalyst for Reduction of 4-Nitrophenol and 2Nitroaniline. Applied Catalysis B Environmental. 158: 129-135.

[15] Gupta, V.K., Atar, N., Yola, M.L., Üstündağ, Z., Uzun, L. (2014). A Novel Magnetic Fe@ Au Core-Shell Nanoparticles Anchored Graphene Oxide Recyclable Nanocatalyst for the Reduction of Nitrophenol Compounds. Water Research. 48: 210-217.

[16] Gupta, V.K., Yola, M.L., Eren, T., Kartal, F., Çağlayan, M.O., Atar, N. (2014). Catalytic Activity of Fe@ Ag Nanoparticle Involved Calcium Alginate Beads for the Reduction of Nitrophenols. Journal of Molecular Liquids. 190: 133-138.

[17] Ma, H., Wang, H., Wu, T., Na, C. (2016). Highly Active Layered Double HydroxideDerived Cobalt Nano-Catalysts for $p$ Nitrophenol Reduction. Applied Catalysis B Environmental. 180: 471-479.

[18] Nasrollahzadeh, M., Sajadi, S.M., RostamiVartooni, A., Bagherzadeh, M., Safari, R. (2015). Immobilization of Copper Nanoparticles on Perlite: Green Synthesis, Characterization and Catalytic Activity on Aqueous Reduction of 4-Nitrophenol. Journal of Molecular Catalysis A Chemical. 400: 22-30.

[19] Du, X.Y., He, J., Zhu, J., Sun, L.J., An, S.S. (2012). Ag-Deposited Silica-Coated $\mathrm{Fe}_{3} \mathrm{O}_{4}$ Magnetic Nanoparticles Catalyzed Reduction of p-Nitrophenol. Applied Surface Science. 258(7): 2717-2723.

[20] Kaniyoor, A., Baby, T.T., Ramaprabhu, S. (2010). Graphene Synthesis via Hydrogen Induced Low Temperature Exfoliation of Graphite Oxide. Journal of Materials Chemistry. 20(39): 8467-8469. 
[21] Wang, H., Maiyalagan T., Wang X. (2012). Review on Recent Progress in Nitrogen-Doped Graphene: Synthesis, Characterization, and Its Potential Applications. ACS Catalysis. 2(5): 781-794.

[22] Wei, D., Liu, Y., Wang, Y., Zhang, H., Huang, L., Yu, G. (2009). Synthesis of N-Doped Graphene by Chemical Vapor Deposition and Its Electrical Properties. Nano Letters. 9(5): 1752-1758.

[23] Panchakarla, L.S., Subrahmanyam, K.S., Saha, S.K., Govindaraj, A., Krishnamurthy, H.R., Waghmare, U.V., Rao, C.N.R. (2009). Synthesis, Structure, and Properties of Boron-and Nitrogen-Doped Graphene. Advanced Materials. 21(46): 4726-4730.

[24] Tsai, C.W., Tu, M.H., Chen, C.J., Hung, T.F., Liu, R.S., Liu, W.R., Shy, D.S. (2011). Nitrogen-Doped Graphene Nanosheet-Supported Non-Precious Iron Nitride Nanoparticles as an Efficient Electrocatalyst for Oxygen Reduction. RSC Advances. 1(7): 1349-1357.

[25] Van Dommele, S., Romero-Izquirdo, A., Brydson, R., De Jong, K.P., Bitter, J.H. (2008). Tuning Nitrogen Functionalities in Catalytically Grown Nitrogen-Containing Carbon Nanotubes. Carbon. 46(1): 138-148.
[26] Arrigo, R., Hävecker, M., Schlögl, R., Su, D.S. (2008). Dynamic Surface Rearrangement and Thermal Stability of Nitrogen Functional Groups on Carbon Nanotubes. Chemical Communications. 40: 4891-4893.

[27] Chunlei, W., Ding, M., Xinhe, B. (2009). Carbon Nanomaterials and their Heterogeneous Catalytic Application. Progress in Chemistry. 21(9): 1705-1721.

[28] Gómez-Navarro, C., Weitz, R.T., Bittner, A.M., Scolari, M., Mews, A., Burghard, M., Kern, K. (2007). Electronic Transport Properties of Individual Chemically Reduced Graphene Oxide Sheets. Nano Letters. 7(11): 3499-3503.

[29] Zhao, Z., Ge, G., Li, W., Guo, X., Wang, G. (2016). Modulating the Microstructure and Surface Chemistry of Carbocatalysts for Oxidative and Direct Dehydrogenation: A Review. Chinese Journal of Catalysis. 37(5): 644-670. 